\title{
Long non-coding RNA PICART1 suppresses proliferation and promotes apoptosis in lung cancer cells by inhibiting JAK2/STAT3 signaling
}

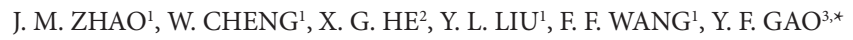 \\ ${ }^{1}$ Department of Respiratory Medicine, The Affiliated Hospital of Qingdao University, Qingdao 266003, China; ${ }^{2}$ Department of Respiratory \\ Medicine, People's Hospital of Rizhao Lanshan, Rizhao 276807, China; ${ }^{3}$ Department of President's Office, The Affiliated Hospital of Qingdao \\ University, Qingdao 266003, China
}

*Correspondence: yufanggao0038@126.com

Received November 30, 2017 / Accepted April 4, 2018

\begin{abstract}
Lung cancer remains the most common cause of tumor-related death worldwide. Recent studies have revealed that long non-coding RNAs (lncRNAs) are involved in the development of various cancers, including lung cancer. This study investigates the molecular basis and effect of lncRNA PICART1 on lung cancer. We first assessed the PICART1 expression in lung cancer in vitro and in vivo by qRT-PCR. Then the expression of PICART1 in SPC-A-1 and NCI-H1975 cell lines was inhibited and over-expressed by transient transfections. Thereafter, cell viability, cell cycle, migration and apoptosis were measured by MTT, Transwell and flow cytometry assay. qRT-PCR and western blot analysis were then performed to assess the expression levels of apoptosis- and migration-related proteins and the JAK2/STAT3 pathway proteins. Tumor formation was measured by xenograft tumor model assay in vivo. PICART1 expression was down-regulated in human lung cancer tissues and cell lines. While PICART1 knockdown increased cell viability of lung cancer cell lines, its over-expression inhibited cell cycle progression and promoted apoptosis in SPC-A-1 and NCI-H1975 cell lines. PICART1 over-expression also inhibited migration in up-regulation of E-cadherin, and down-regulation of Twist1, MMP2 and MMP9. We also found that PICART1 inhibition can regulate cell apoptosis and migration by activating the JAK2/STAT3 pathway, and in vivo experiments revealed that PICART1 knockdown significantly promoted tumor formation. This study demonstrates that PICART1 over-expression has an anti-growth and anti-metastasis role in lung cancer cells and its tumor suppression may be via regulation of the JAK2/STAT3 pathway.
\end{abstract}

Key words: lung cancer, PICART1, apoptosis, migration, JAK2/STAT3 pathway

Lung cancer is the most common malignant neoplasms worldwide and is associated with highest incidence, morbidity and mortality [1]. More than $80 \%$ of all lung cancers consist of non-small cell lung cancer (NSCLC) [2]. While surgery, radiation therapy and chemotherapy are established lung cancer treatment [3], the long-term survival rate is still very low because of tumor metastasis [4]. It is therefore essential to obtain a better understanding of molecular biology to develop more effective and novel therapies.

Current research has revealed that epigenetic abnormalities have crucial roles in human cancer development and progression [5]. Genetic continuity involves epigenetic regulation, such as histone deacetylation, DNA methylation and the regulation of non-coding RNA (ncRNA) [6, 7]. Long non-coding RNAs (lncRNAs) are a class of newfound non-coding RNAs greater than 200 nucleotides in length with crucial functional importance in governing funda- mental biological processes and diseases [8]. Increasing evidence indicates that lncRNAs have pivotal roles in cellular development, differentiation, the immune response and many other biological processes [9-11]. Moreover, lncRNA deregulation has been implicated in various types of human cancers including lung [12-14], gastric [11], colorectal [15] and others [16]. p53-inducible cancer-associated RNA transcript 1 (PICART1), a novel lncRNA transcript, $2.53 \mathrm{~kb}$ in length, was first found decreased in breast and colorectal cancer cells and in tissues where they inhibited cell proliferation and migration [17]. It may also function as a tumor suppressor in the AKT GSK3 $\beta / \beta$-catenin signalling cascade in breast and colorectal cancer cells. However, its biological role and regulatory mechanism in the development of lung cancer remains unclear.

Herein, we investigated PICART1 function in the growth and metastasis of lung cancer cells and its underlying mecha- 
nisms. We found that its expression decreased in lung cancer tissues and cell lines compared with controls, and that PICART1 knockdown increased cell viability and its overexpression reduced cell viability and migration, arrested more cells in the G0/G1 phase and promoted cell apoptosis. Furthermore, its underlying regulation mechanism is considered to involve inhibition of the JAK2/STAT3 pathway. Finally, in vivo experiments further displayed that PICART1 knockdown promoted lung cancer tumor formation. This provides new insight into the PICART1's molecular function and regulatory mechanisms in lung cancer pathogenesis.

\section{Materials and methods}

Cell culture and the collection of human lung cancer tissues. Five human lung cancer cell lines, A549, SPC-A-1, NCI-H358, NCI-H1975 and HCI-H292 cells and the normal lung epithelial cells, BEAS-2B, were obtained from the American Type Culture Collection (ATCC, Rockville, MD, USA). All cells were cultured at Roswell Park Memorial Institute (RMPI)-1640 (Hyclone, Logan, UT, USA) supplemented with $10 \%$ fetal bovine serum (FBS, Hyclone) and 1\% penicillin-streptomycin solution (Gibco, Life Technologies, Grand Island, $\mathrm{NY}$ ) at $37 \mathrm{C}$ in $5 \% \mathrm{CO}_{2}$.

Lung cancer tissues and corresponding adjacent lung tissues were obtained from 30 pairs of patients $(17$ males and 13 females, aged from 30-60 years) who underwent surgery at the Affiliated Hospital of Qingdao University from February 2011 to May 2015. The study protocol had informed patient consent, was approved by the ethics committee of the Affiliated Hospital of Qingdao University and all patients had no pre-operative neo-adjuvant treatment The specimens were preserved in liquid nitrogen immediately for subsequent testing. The specimen collection procedure was performed with informed patient consent and approval of the Medical Ethics Committee. All research was carried out in accordance with the provisions of the 1975 Helsinki Declaration.

Transfection and generation of stably transfected cell lines. Full-length PICART1 sequences and short-hairpin RNA directed against PICART1 were constructed in pEX-2 and U6/GFP/Neo plasmids (GenePharma, Shanghai, China), respectively. These were referred as to pEX-PICART1 and sh-PICART1. The plasmids carrying non-targeting sequences were used as negative controls (NC) of pEX-PICART1 and sh-PICART1 and are referred as to pEX and sh-NC. The lipofectamine 3000 reagent (Life Technologies Corporation, Carlsbad, CA, USA) was used for the cells transfection according to the manufacturer's instructions.

MTT assay. Cell viability was evaluated by 3-(4, 5-dimethylthiazol-2-yl)-2, 5-diphenyltetrazolium bromide (MTT) colorimetric assay (Sigma-Aldrich, St Louis, MO, USA) according to the manufacturer's instructions. Cells infected with pEX-PICART1 and sh-PICART1 were collected and seeded in 96-well plates at $5 \times 10^{3}$ cells/well. After days 1, 2,
3 and $4, \operatorname{MTT}(5 \mathrm{mg} / \mathrm{ml})$ was added to the cells in the dark and incubated for further 4 hours at $37^{\circ} \mathrm{C}$. Supernatant was removed and $100 \mu \mathrm{l}$ dimethylsulfoxide (DMSO, SigmaAldrich) was added to each well to dissolve the cells. The optical density of each well was measured at $570 \mathrm{~nm}$ on a microplate reader (Bio-Rad Laboratories, Orlando, FL, USA).

Quantitative real time RT-PCR (qRT-PCR) analysis. Total RNA was extracted by TRIzol reagent (Invitrogen, CA, USA), and was reverse transcribed into complementary DNA (cDNA) using a Reverse Transcription Kit (Takara, Dalian, China) according to the manufacturer's instructions. PICART1 expression was determined by qRT-PCR using the SYBR Green Master Mix (Takara). PICART1 primers are as follows: forward 5'-AGG CAG CTA CTG TAA TAA T-3' and reverse 5'-GTA CCC TGG GCC TTT CTT AC-3'. GAPDH level was used as internal control and relative expression changes were calculated using the $2^{-\Delta \Delta \mathrm{Ct}}$ method.

Western blot. The protein used for Western blot was extracted using radio-immuno-precipitation assay (RIPA) lysis buffer (Beyotime Biotechnology, Shanghai, China) supplemented with protease inhibitors (Roche, Basel, Switzerland). The protein concentration was quantified using the BCA Protein Assay Kit (Pierce, Rockford, IL, USA). Western blot was established in the Bio-Rad Bis-Tris Gel system and primary antibodies were: anti-Twist1 (\#46702), anti-MMP2 (\#87809), anti-MMP9 (\#13667), anti-E-cadherin (\#3195), anti-Bcl-2 (\#4223), anti-Bax (\#5023), anti-caspase-3 (\#9662), anti-p-STAT3 (\#9145), anti-STAT3 (\#12640), antip-JAK2 (\#4406), anti-JAK2 (\#3230), anti-Cyclin D1 (\#2978), anti-P21 (\#2947) and anti-GAPDH (\#2118). These were all purchased from Cell Signalling Technology (Beverly, MA, USA) and prepared in 5\% bovine serum albumin (BSA, Sigma-Aldrich) at 1:1000 dilution. The blots were incubated with primary antibodies overnight at $4^{\circ} \mathrm{C}$, followed by washing and incubation with secondary antibody marked by horseradish peroxidase for 1 hour at room temperature. After rinsing, the blots were developed with ECL solution (Pierce) and visualized by Image Lab $^{\mathrm{TM}}$ Software (Bio-Rad Laboratories).

Cell cycle distribution analysis. Cells infected with pEX-PICART1 and sh-PICART1 were collected and washed twice with cold phosphate buffered saline (PBS) and fixed in $70 \%$ ethanol. Fixed cells were washed in PBS and incubated with $1 \mathrm{mg} / \mathrm{ml}$ RNase A and then stained with $0.1 \mathrm{mg} / \mathrm{ml}$ propidium iodide (PI, Sigma-Aldrish) for $30 \mathrm{~min}$ at $37^{\circ} \mathrm{C}$. Intensities of fluorescence signals were measured by flow cytometer (Beckman Coulter, Fullerton, CA, USA). The percentage of cells in the G0/G1, S and G2/M phases was determined using FlowJo software (version 7.6.1, Tree Star, Ashland, OR, USA).

Apoptosis assay. Apoptosis analysis identified and quantified apoptotic cells by Annexin V-PE apoptosis detection kit (Beijing Biosea Biotechnology, Beijing, China). Cells were harvested and rinsed twice with cold PBS and then re-suspended in $500 \mu \mathrm{l} / 1$ binding buffer and mixed with $5 \mu \mathrm{l}$ 
Annexin $\mathrm{V}$ and $5 \mu \mathrm{l} \mathrm{PE}$ for 15 minutes at room temperature in the dark. Treated cells were washed twice with cold PBS and re-suspended in buffer. Flow cytometry analysis was via FACS can (Beckman Coulter, Inc., Fullerton, CA, USA) and data was analysed by FlowJo software (Tree Star Inc. Ashland, OR, USA).

Migration assay. The Transwell (BD Biosciences, San Jose, CA, USA) migration assay with $8 \mu \mathrm{m}$ pore inserts analyzed SPC-A-1 and NCI-H1975 cell migration ability. In brief, transfected SPC-A-1 and NCI-H1975 cells were washed twice with PBS, suspended in $200 \mu \mathrm{l}$ serum-free culture medium and added to the upper chamber. The lower chamber was then supplemented with $600 \mu \mathrm{l}$ of complete medium containing 10\% FBS (Hyclone). After incubation for 24 hours at $37^{\circ} \mathrm{C}$, the cells on the lower side were washed with PBS and fixed with $4 \%$ methanol for 30 minutes (NIST, USA) Non-migrated cells on the upper surface of the filter were removed with a wet cotton swab, and migrated cells were stained with $0.1 \%$ crystal violet for 20 minutes (Merck, Darmstadt, Germany). Finally, the cells were counted under a microscope (Leica Microsystems, Watzlar, Germany).

Wound healing scratch assay. SPC-A-1 and NCI-H1975 cells were grown in 6-well plate and incubated to confluence. A $10 \mu$ pipette tip made scratches to mimic wounds. The transfected cells were washed thrice with PBS and added to serum-free medium. After incubation for 48 hours under the conventional culture conditions, the area of migrated cells in the scratch was measured.

Tumor formation assay in vivo. The nude mice (9-12 weeks old) were obtained from Shanghai experimental animal center of the Chinese Academy of Sciences (Shanghai, China), and the mice were housed in laminar airflow cabinets under specific pathogen-free conditions. SPC-A-1 cells were harvested from culture flasks and transferred to serum-free PBS (PAA Laboratories $\mathrm{GmbH}$ ) and cell suspensions were then injected subcutaneously into mice neck, and left and right shoulders. Tumor size was obtained by standard caliper measuring the tumor length and width in a blinded fashion, and tumor volume was then calculated. The mice were sacrificed 30 days later by cervical dislocation in deep $\mathrm{CO}_{2}$ anaesthesia. The primary tumors were surgically removed and tumor weight was determined.

Statistical analysis. All experiments were in triplicate and results are presented as the mean \pm standard deviation (SD). Statistical analysis was by Graphpad 6.0 statistical software (GraphPad Software, San Diego, CA, USA), and p-values were calculated by one-way analysis of variance (ANOVA). p-value $<0.05$ was statistically significant.

\section{Results}

PICART1 was down-regulated in human lung cancer tissues and cell lines. qRT-PCR analysis showed that PICART1 expression was markedly down-regulated in human lung tumor tissue compared to adjacent lung tissue $(\mathrm{p}<0.001)$ (Figure 1A). We also examined the expression levels of PICART1 in five lung cancer cell lines and BEAS-2B cells. Figure 1B highlights that PICART1 expression was significantly reduced in five lung cancer cell lines compared with BEAS- $2 B$ cells $(p<0.05, p<0.01$, or $p<0.001)$, and the lowest expression of PICART1 was detected in SPC-A-1 and NCI-H1975 cell lines.

PICART1 knockdown promoted in vitro lung cancer cell viability. We infected SPC-A-1 and NCI-H1975 cells with PICART1 silencing vector (sh-PICART1) to knockdown the PICART1 expression and characterize the functional impor-
A

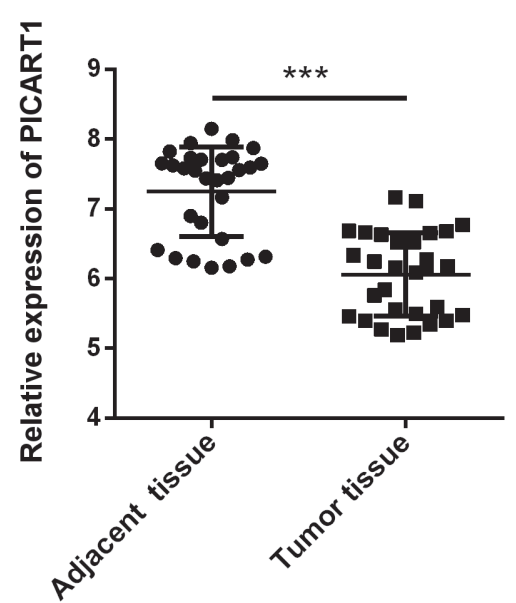

B

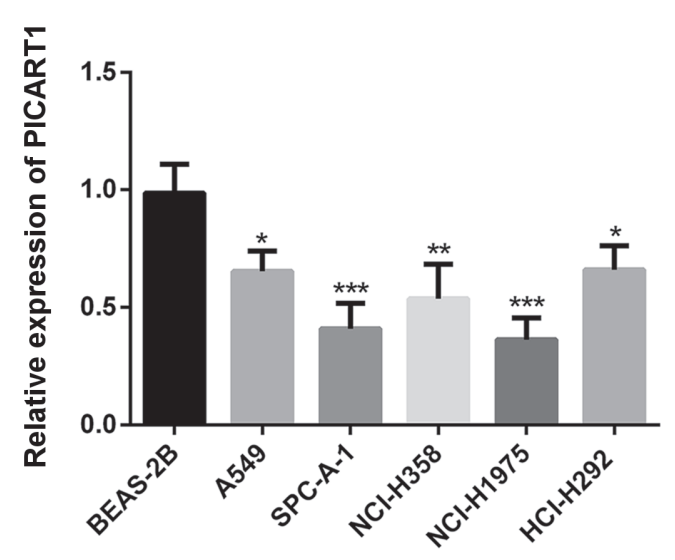

Figure 1. PICART1 down-regulated in human lung cancer tissues and cell lines. A) PICART1 mRNA level was significantly lower in human lung cancer tissues than in adjacent-normal lung tissues $(n=30)$. B) PICART1 mRNA levels in five human cancer cell lines and BEAS-2B cells were significantly lower in lung cancer cell lines compared with BEAS-2B cells, and the lowest expression of PICART1 was detected in SPC-A-1 and NCI-H1975 cell lines. ${ }^{*} \mathrm{p}<0.05,{ }^{* *} \mathrm{p}<0.01,{ }^{* * *} \mathrm{p}<0.001$. 
tance of PICART1 in lung tumorigenesis. qRT-PCR assay examined the efficacy of transfection. As expected, PICART1 expression was significantly reduced by PICART1 silencing in SPC-A-1 and NCI-H1975 cells ( $\mathrm{p}<0.001)$ (Figures 2A and 2B). Next, we detected the role of PICART1 in lung cancer cell viability, and MTT assay analysis showed that PICART1 knockdown remarkably increased SPC-A-1 and NCI-H1975 cell viability after the 3rd and 4 th days $(\mathrm{p}<0.05$, or $\mathrm{p}<0.01)$ (Figures 2C and 2D).

Over-expression of PICART1 induced cell cycle arrest in G0/G1 phase and apoptosis in lung cancer cells. We then further over-expressed the PICART1 level in SPC-A-1 and NCI-H1975 cells through transfection with PICART1 expressing-vector to further investigate the role of PICART1 in the lung cancer cell cycle and apoptosis. Figures $3 \mathrm{~A}$ and $\mathrm{B}$ show that the expression of PICART1 was markedly up-regulated in the two lung cancer cell lines after transfection with pEX-PICART1 $(\mathrm{p}<0.001)$. FACScan analysis showed that over-expression of PICART1 reduced the rate of G2-M and S phase cells and increased the rate of G0-G1 phase cells in both cell lines (Figures $3 \mathrm{C}$ and D). Figures $3 \mathrm{E}$ and $3 \mathrm{~F}$ then show that Annexin V-PE staining assay indicated that PICART1 over-expression in SPC-A-1 and NCI-H1975 cells significantly elevated cell apoptotic rates compared to $\mathrm{pEX}$ $(\mathrm{p}<0.01)$. This indicates that over-expression of PICART1 arrests SPC-A-1 and NCI-H1975 cells in the G0/G1 phase and induces apoptosis.

Knockdown of PICART1 promoted cell migration of lung cancer cells in vitro. The PICART1 over-expressing and silencing vectors were transfected into SPC-A-1 and NCI-H1975 cells to determine the PICART1 influence on cell migration. Tranwell migration assay then analyzed SPC-A-1
A

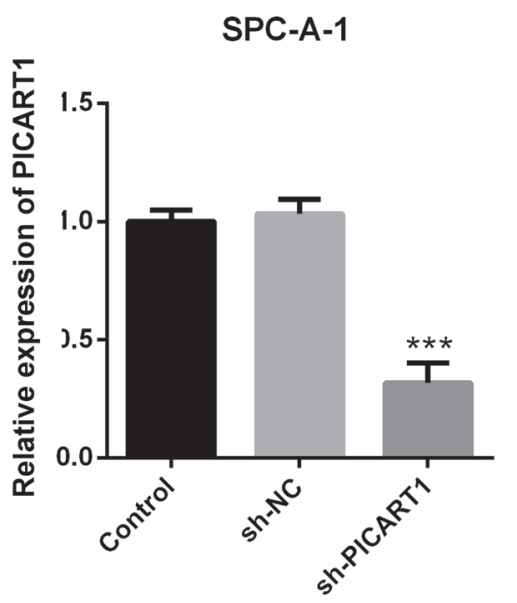

C

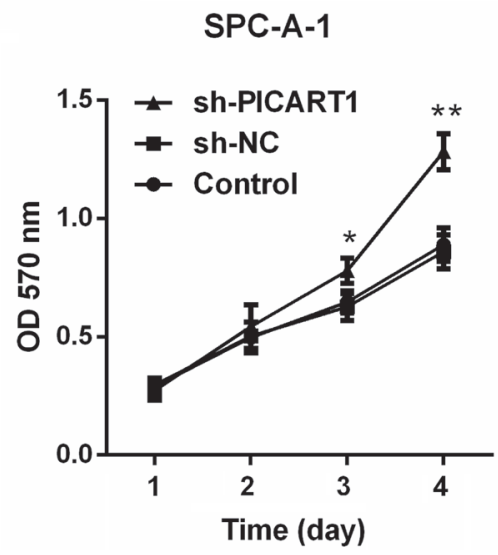

B

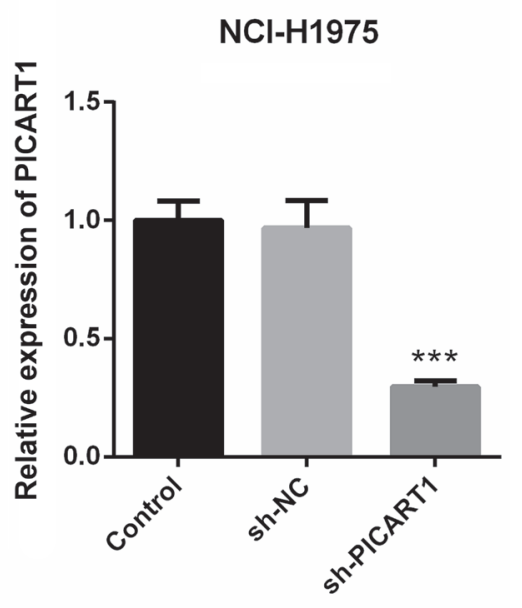

D

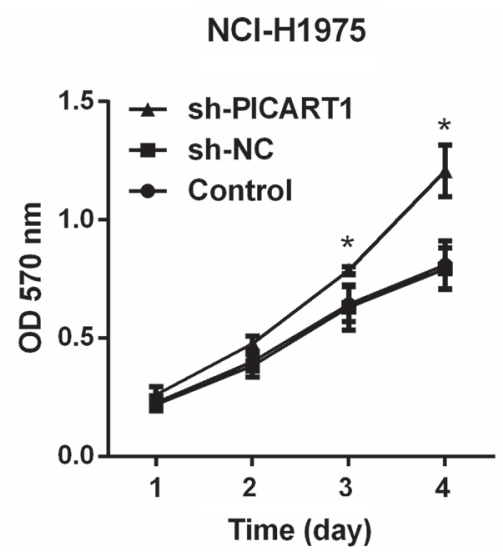

Figure 2. Knockdown of PICART1 promoted cell viability of lung cancer cells in vitro. Knockdown of PICART1 by shRNA transfection showed notably expression inhibited in mRNA levels in A) SPC-A-1 and B) NCI-H1975 cells. Cell viability was measured by MTT assay in SPC-A-1 (C) and NCI-H1975 (D) cells transfected sh-NC or sh-PICART1. PICART1 knockdown significantly increased cell viability in both cell lines after 3 and 4 day. ${ }^{\star}$ p $<0.05$, ${ }^{* *} \mathrm{p}<0.01,{ }^{* * *} \mathrm{p}<0.001$. 
A

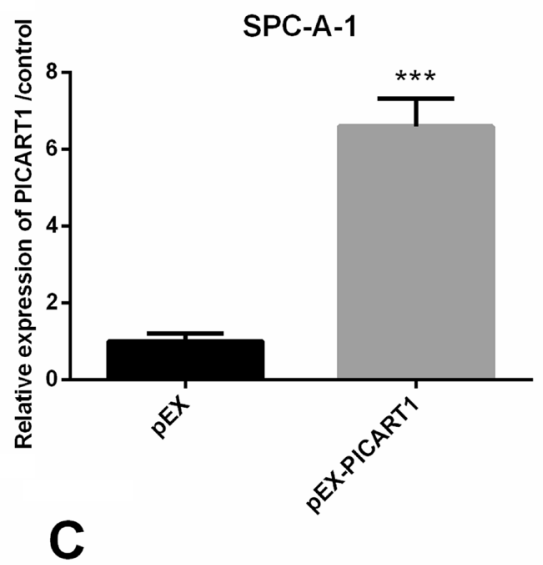

SPC-A-1

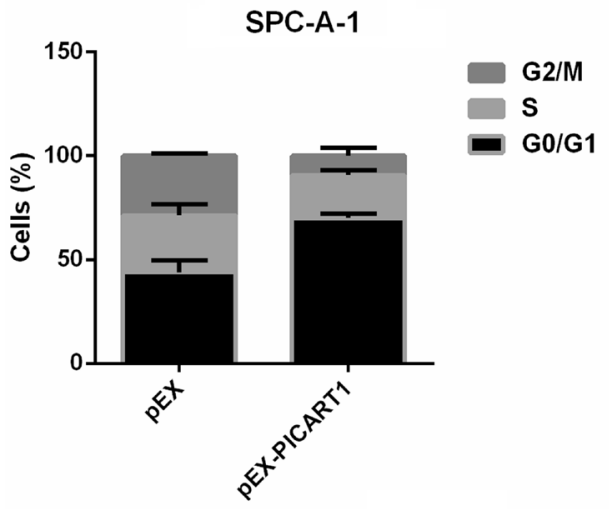

$\mathbf{E}$

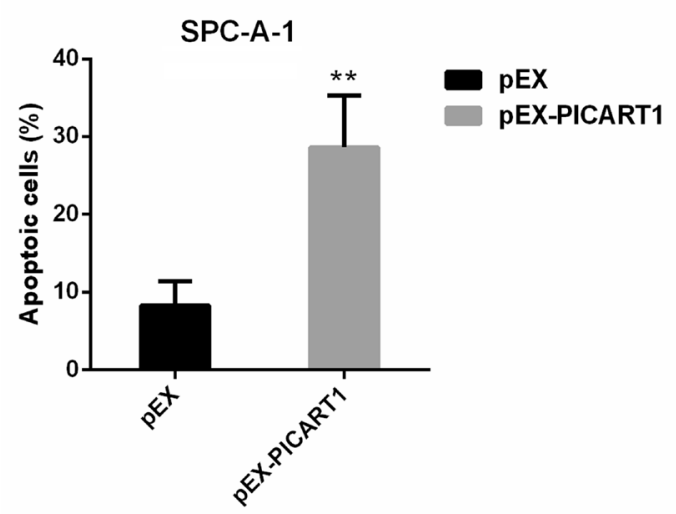

B

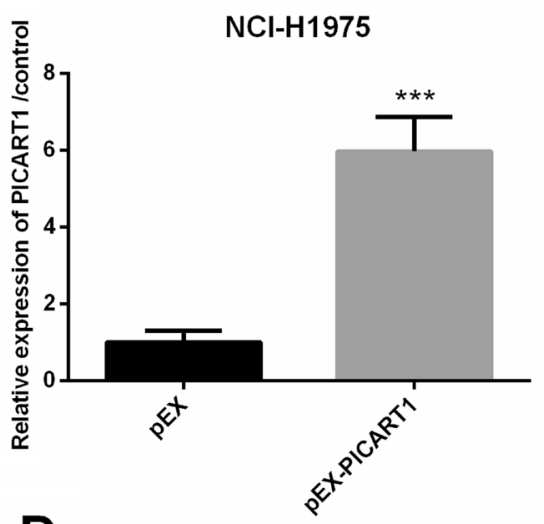

D

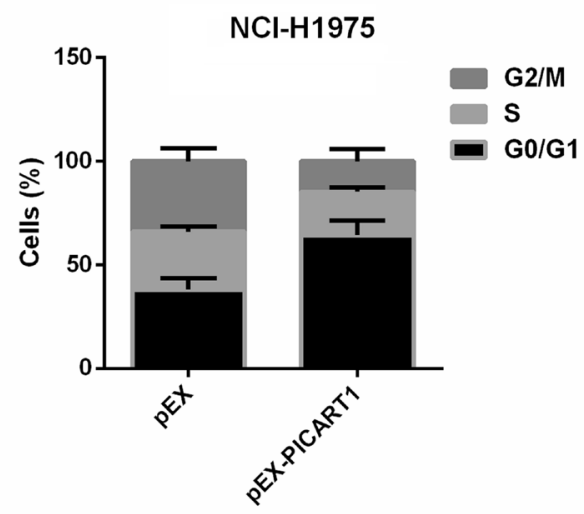

$\mathbf{F}$

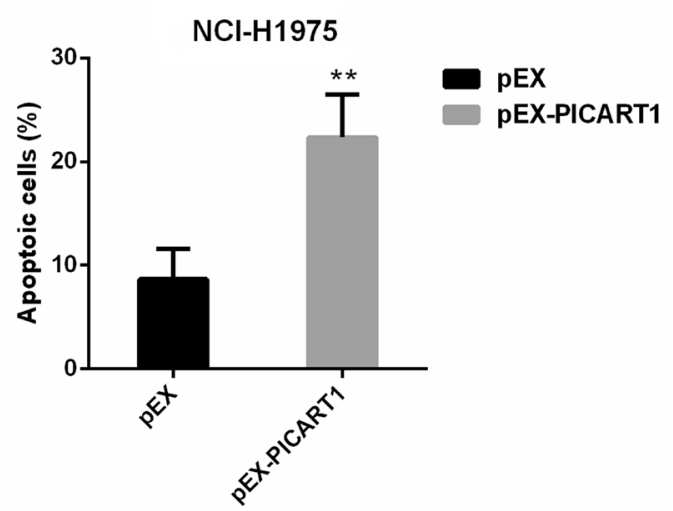

Figure 3. Overexpression of PICART1 induced cell cycle arrest in G0/G1 phase and apoptosis in lung cancer cells. Overexpression of PICART1 by transfection with PICART1 overexpressing vector showed notably expression promoted in mRNA levels in SPC-A-1 (A) and NCI-H1975 (B) cells. Cell cycle arrest was analyzed using flow cytometry in SPC-A-1 (C)and NCI-H1975 (D) cells, and PICART1 overexpression reduced the rate of G2/M and $\mathrm{S}$ phase cells and increased the rate of G0/G1 phase cells in both cell lines. Cell apoptosis was analyzed by Annexin V/PE staining in SPC-A-1 (E) and NCI-H1975 (F) cells, and PICART1 overexpression significantly elevated cell apoptotic rates in comparison with $\mathrm{pEX} .{ }^{* *} \mathrm{p}<0.01,{ }^{* * *} \mathrm{p}<0.001$.

and NCI-H1975 cell migration ability. Figures 4A and B show that PICART1 knockdown significantly enhanced cell migration in both SPC-A-1 and NCI-H1975 cells $(\mathrm{p}<0.01)$ and over-expression produced the opposite effect $(\mathrm{p}<0.05)$. The wound-healing scratch assay results revealed that
PICART1 knockdown remarkably decreased the distance after 12, 24 and 72 hours in both SPC-A-1 and NCI-H1975 cells $(p<0.05)$ (Figures $4 \mathrm{C}$ and $\mathrm{D})$. All these results suggest PICART1 knockdown promotes cell migration in SPC-A-1 and NCI-H1975 cells. 
PICART1 over-expression effect on apoptosis and migration-related protein expression. We further investigated the PICART1 over-expression effect on the expression of the $\mathrm{Bcl} 2, \mathrm{Bax}$ and Caspase 3 core proteins involved in apoptosis, and also the Twist1, MMP-2, MMP-9 and E-cadherin core proteins involved in migration. Figure 5A shows that PICART1 over-expression significantly reduced Bcl 2 mRNA level but increased Bax and Caspase 3 mRNA levels in SPC-A-1 cells $(\mathrm{p}<0.05$, or $\mathrm{p}<0.001)$. Moreover, PICART1 over-expression markedly decreased Twist1, MMP2 and MMP9 mRNA expression and elevated the E-cadherin level $(\mathrm{p}<0.05$, or $\mathrm{p}<0.01)$ (Figure $5 \mathrm{~B})$. Similar results were found in the protein expression levels of these core factors in SPC-A-1 (Figure 5C) and NCI-H1975 cells (Figure 5D). These results indicate that PICART1 overexpression promotes cell apoptosis and inhibits migration by regulating the core proteins in these two processes.

We also used TGF- $\beta(10 \mathrm{ng} / \mathrm{ml})$ to treat SPC-A-1 and NCI-H1975 cells, and examined Twist1, MMP-2, MMP-9 and E-cadherin protein levels with Western blot. Results revealed that Twist1, MMP-2, and MMP-9 protein levels were significantly increased and E-cadherin protein decreased in TGF- $\beta$-treated SPC-A-1 and NCI-H1975 cells However, PICART1 over-expression reversed these results (Figures 5E and F), and this indicates PICART1 involvement in regulating cell migration by mediating Twist1, MMP-2, MMP-9 and E-cadherin expression in TGF- $\beta$-treated lung cancer cells.

Mechanisms of PICART1 exert its functions in lung cancer cell lines. To explore PICART1mechanisms in tumorigenesis, we then investigated whether PICART1 regulated the JAK2/STAT3 signaling pathway. We found that down-regulation of PICART1 promoted JAK2 and STAT3 phosphorylation in SPC-A-1 (Figure 6A) and NCI-H1975 cells (Figure 6B). In contrast, PICART1 over-expression inhibited JAK2/STAT3 signaling pathway activation by suppressing JAK2 and STAT3 phosphorylation in SPC-A-1 (Figure 6C) and NCI-H1975 cells (Figure 6D). Therefore, we hypothesized that PICART1 knockdown reduced cancer cell apoptosis and migration by activating the JAK2/STAT3
A

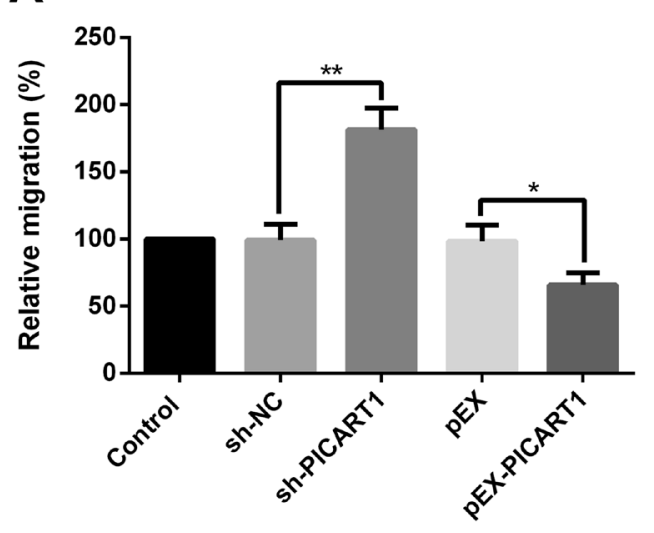

c

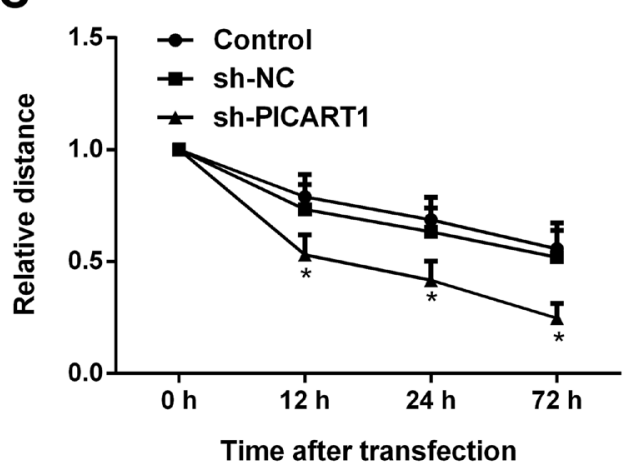

B

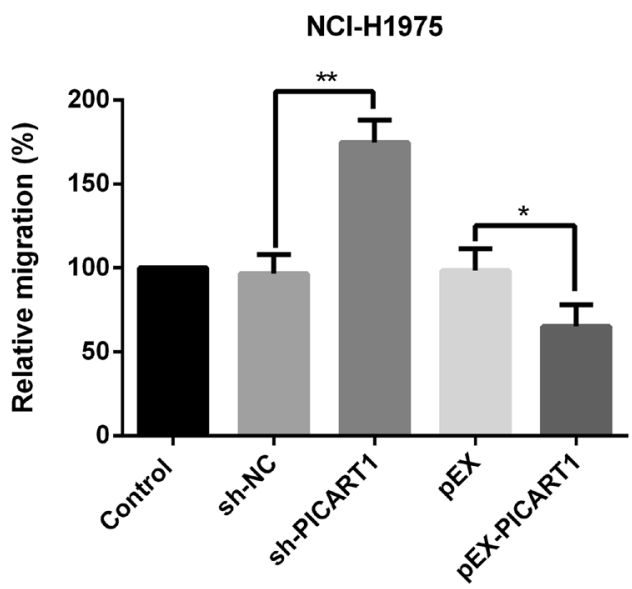

D

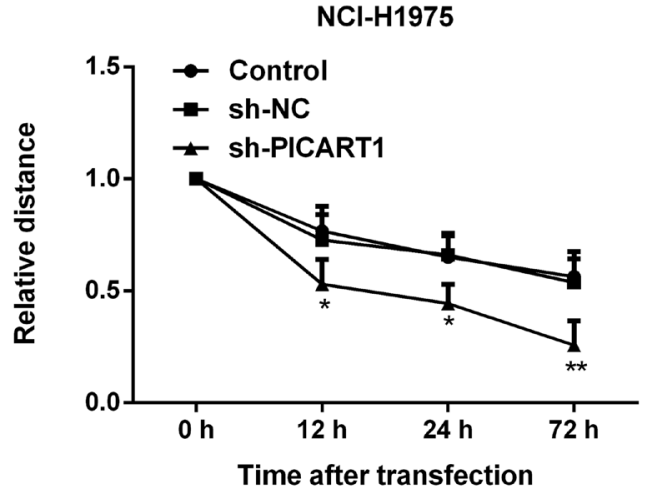

Figure 4. Effect of PICART1 on cell migration in vitro. Cell migration was measured by Transwell migration assay in SPC-A-1 (A) and NCI-H1975 (B) cells transfected sh-NC, sh-PICART1, pEX or pEX-PICART1. PICART1 knockdown significantly increased cell migration in both cell lines. Migration distance was evaluated by scratch wound assay in SPC-A-1 (C) and NCI-H1975 (D) cells transfected sh-NC, sh-PICART1, pEX or pEX-PICART1. PICART1 knockdown significantly decreased the migration distance in both cell lines. ${ }^{*} \mathrm{p}<0.05,{ }^{* *} \mathrm{p}<0.01$. 
signaling pathway. Interesting, we found that the Stattic inhibitor of the JAK2/STAT3 signaling pathway reversed the PICART1 knockdown effect on protein expression and this correlated with apoptosis and migration through decreased Twist1, MMP2, Cyclin D1 and Bcl2 levels and elevated expression of P21, Bax and Caspase 3 in the SPC-A-1 (Figure 6E) and NCI-H1975 cells (Figure 6F) We further investigated whether the PICART1 influence on cell cycle arrest in the G0/G1 phase was also mediated by the JAK2/ STAT3 signaling pathway. The results in Figures 7A and B show that PICART1 knockdown decreased the percentage of SPC-A-1 and NCI-H1975 cells in the G0/G1 phase and this was reversed by JAK2/STAT3 signaling pathway inhibition.

Effect of PICART1 knockdown on tumor formation in vivo. Finally, we explored PICART1 knockdown effect on tumor formation in vivo. Results confirmed that PICART1 knockdown significantly promoted tumor volume after
14-30 day injections compared to controls $(\mathrm{p}<0.05, \mathrm{p}<0.01$ or $\mathrm{p}<0.001$ ) (Figure $8 \mathrm{~A}$ ). We also determined that the tumor weight was markedly enhanced by PICART1 knockdown compared to the control group $(\mathrm{p}<0.01)$ (Figure $8 \mathrm{~B})$. These results highlight PICART1 knockdown can promote tumor formation in vivo.

\section{Discussion}

LncRNAs are known to be involved in multiple key cellular processes; including genomic imprinting, RNA processing, chromatin modification and the modulation of apoptosis and invasion [18-21]. Emerging evidence shows that lncRNAs are essential for the development of malignant lung tumor carcinogenesis, and these have emerged as biomarkers for tumor prediction and diagnosis [7, 22, 23]. For example: (1) lncRNA AGAP2-AS1 can promote the NSCLC
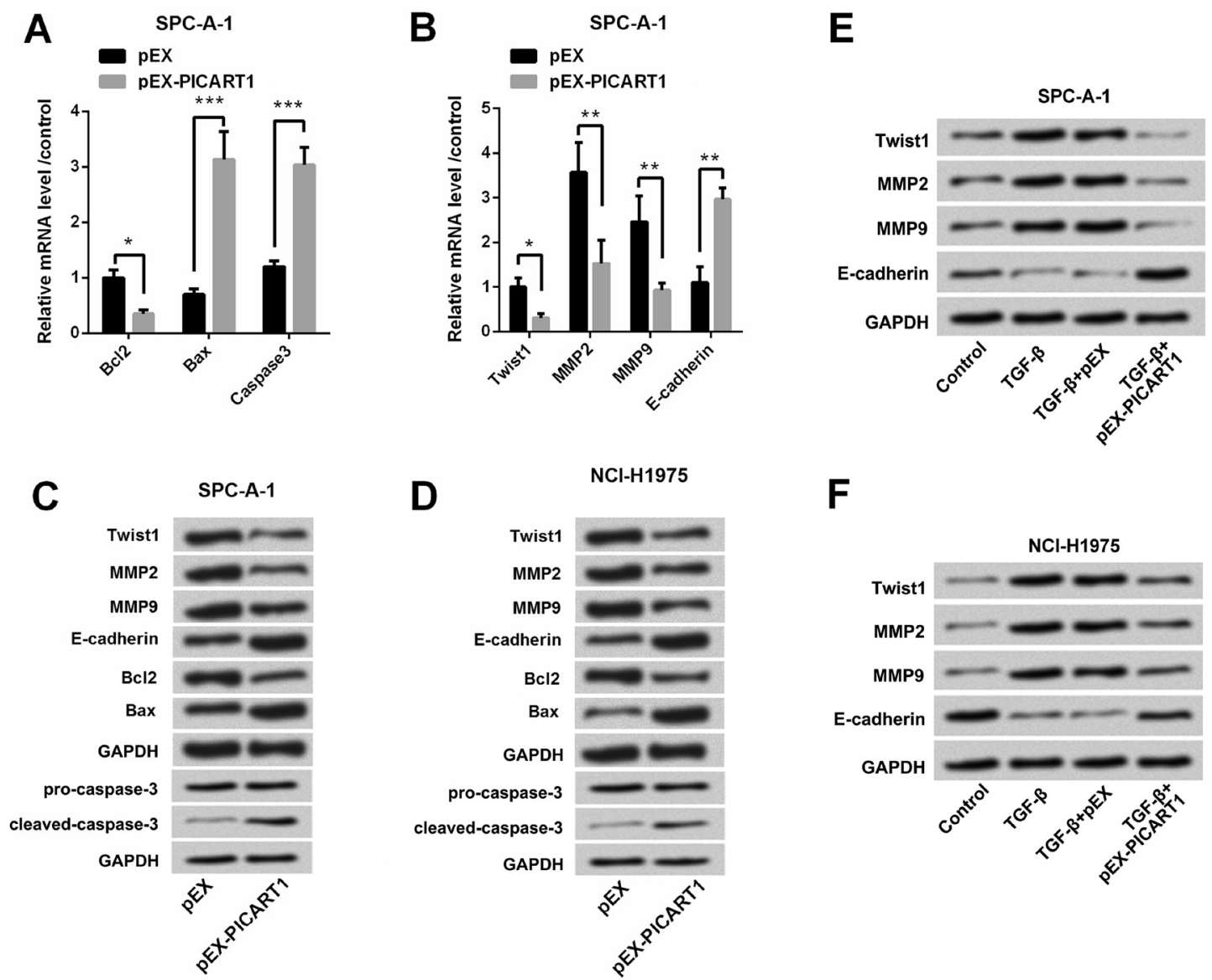

Figure 5. Effect of PICART1 overexpression on apoptosis-related and migration-related protein expressions. SPC-A-1 and NCI-H1975 cells were transfected with pEX or pEX-PICART1. A) RT-PCR analysis identified that PICATR1 overexpression remarkably inhibited Bcl2 expression and induced the expression of Bax and caspase 3 in SPC-A-1 cells. B) RT-PCR analysis identified that overexpression of PICATR1 markedly promoted E-cadherin expression and repressed the expression of Twist1, MMP-2 and MMP-9. Western blot analysis showed that up-regulation of PICART1 reduced the BCL2, Twist1, MMP-2 and MMP-9 levels, while increased the expression of Bax, Caspase3 and E-cadherin in SPC-A-1 (C) and NCI-H1975 (D) cells. The transfected SPC-A-1 and NCI-H1975 cells were stimulated with TGF-u $(10 \mathrm{ng} / \mathrm{ml})$ for $12 \mathrm{~h}$. Western blot analysis showed that the regulation effect of PICART1 overexpression on Twist1, MMP-2, MMP-9 and E-cadherin levels were obviously reversed after adding TGF- $\beta$ in both SPC-A-1 (E) and NCI-H1975 (F) cells. ${ }^{*} \mathrm{p}<0.05,{ }^{* *} \mathrm{p}<0.01,{ }^{* * *} \mathrm{p}<0.001$. 


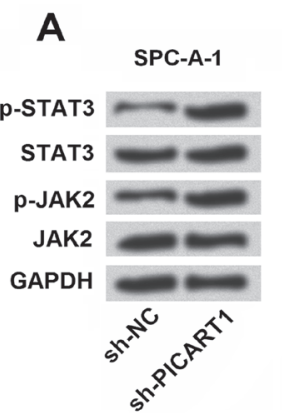

B
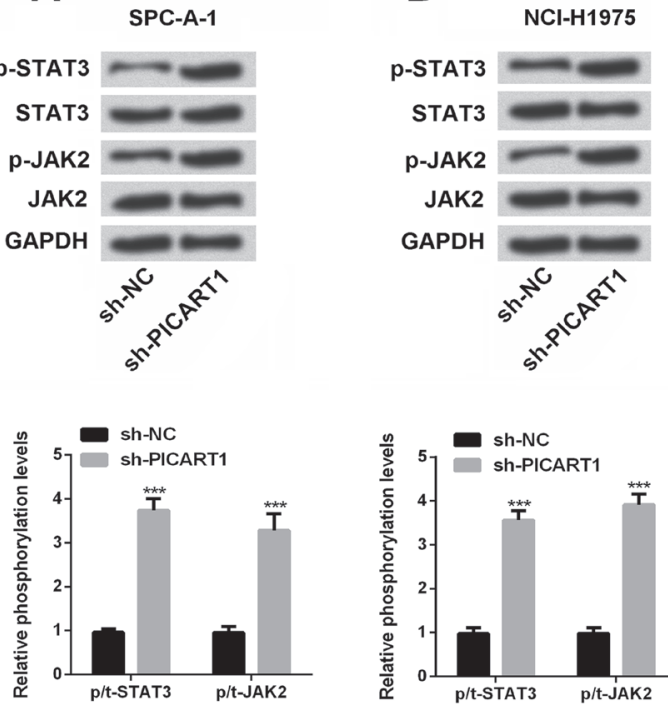

C
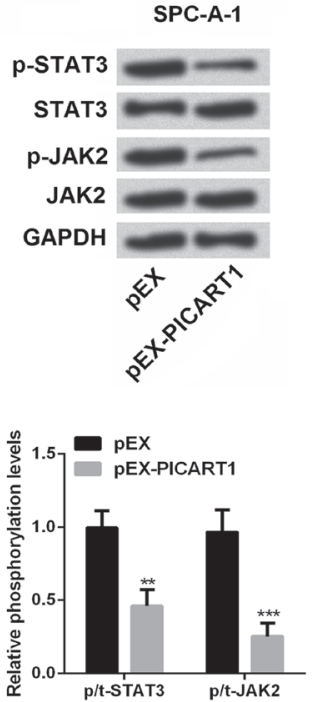

D
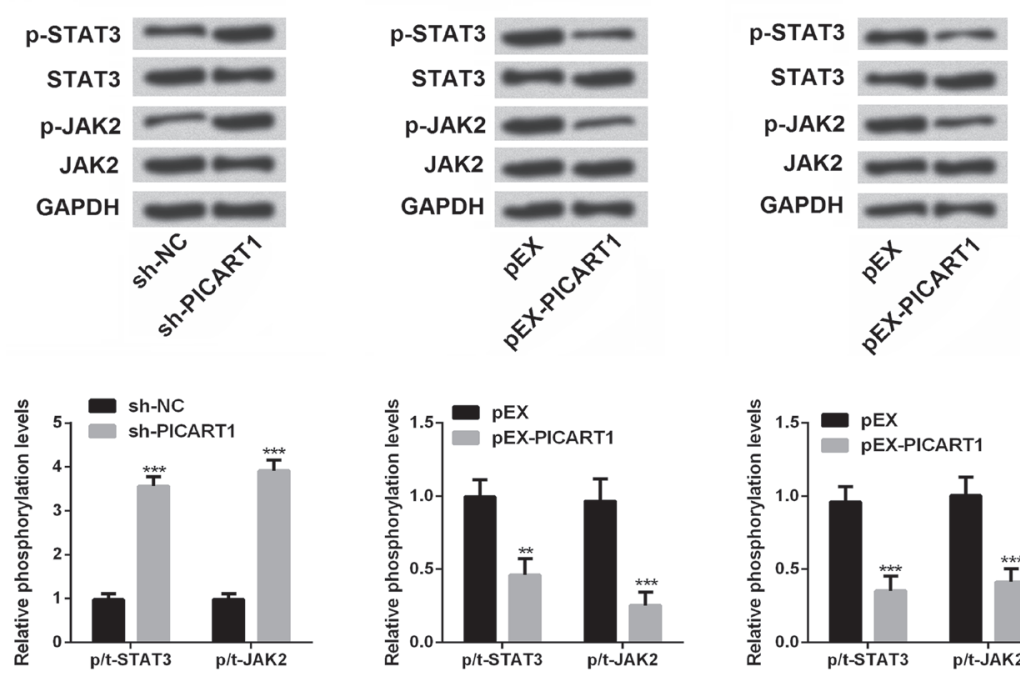

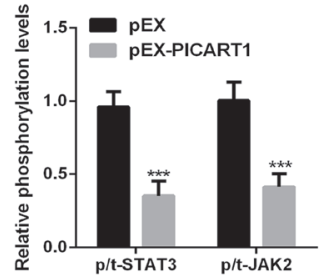

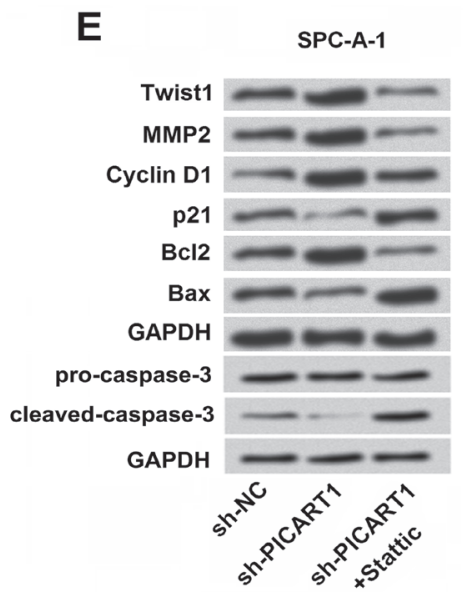

$\mathbf{F}$

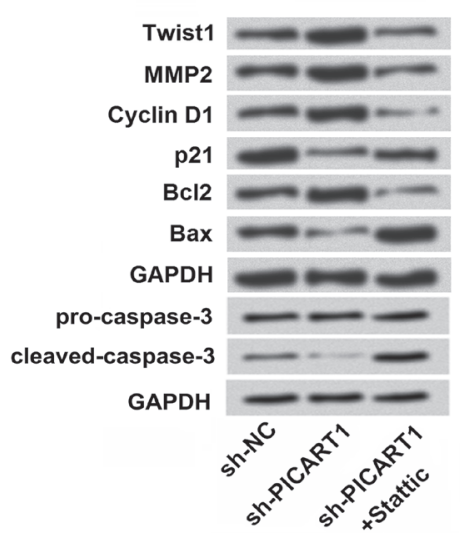



\#\#\#\# compared to thesh-PICART1 group

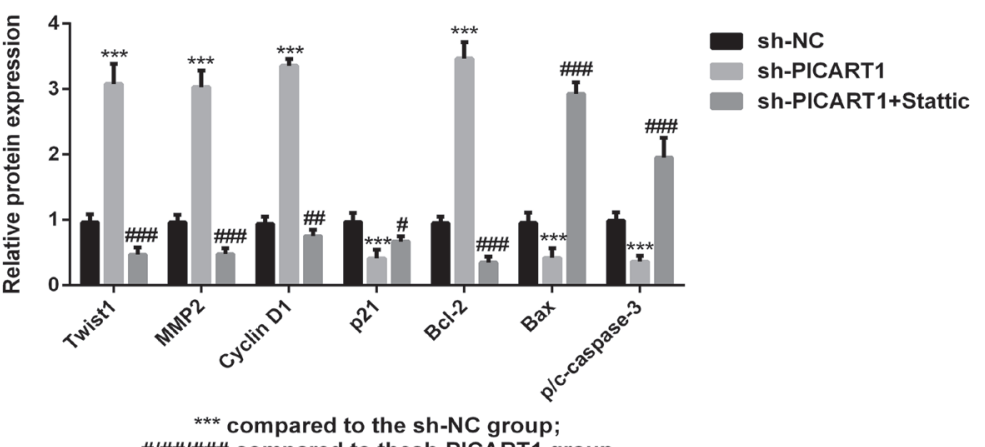

Figure 6. Mechanisms of PICART1 exert its functions in lung cancer cell lines. SPC-A-1 and NCI-H1975 cells were transfected with sh-PICART1, pEXPICART or their corresponding controls, sh-NC and pEX. Western blot analysis showed that PICART1 knockdown induced activation of STAT3/JAK2 pathway effectors in SPC-A-1 (A) and NCI-H1975 (B) cells. PICART1 overexpression suppressed the activation of p-STAT3/JAK2 pathway effectors in SPC-A-1 (C) and NCI-H1975 (D) cells. The inhibitor of p-STAT3/JAK2 pathway, Stattic, blocked the regulation of PICART knockdown on migration and apoptosis correlated factors expression in SPC-A-1 (E) and NCI-H1975 (F) cells. 
A

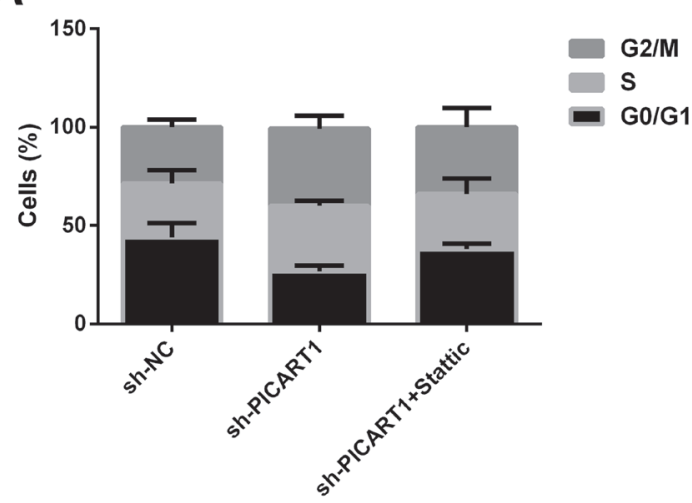

B

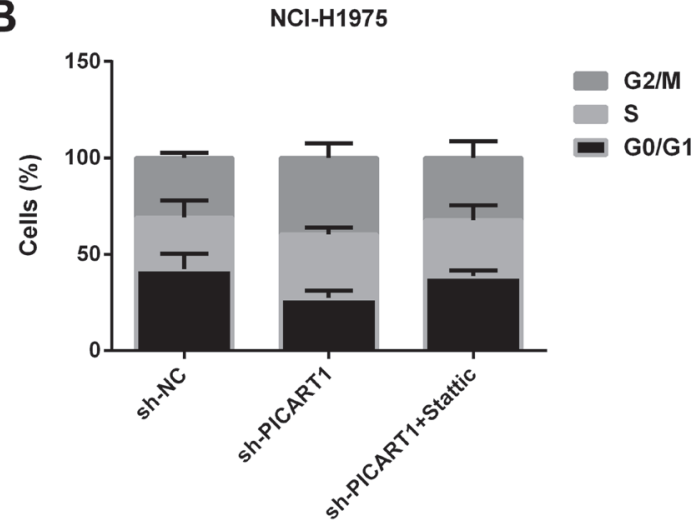

Figure 7. Effect of PICART1 on cell cycle arrest in G0/G1 phase was mediated by JAK2/STAT3 signaling pathway. SPC-A-1 cells were transfected with sh-PICART1 or sh-NC. Cell cycle arrest was analyzed using flow cytometry in SPC-A-1 (A) and NCI-H1975 (B) cells, and PICART1 knockdown reduced the rate of G0/G1 phase cells in both cell lines, but the inhibitory effect was reversed by the inhibitor of JAK2/STAT3 signaling pathway.

A

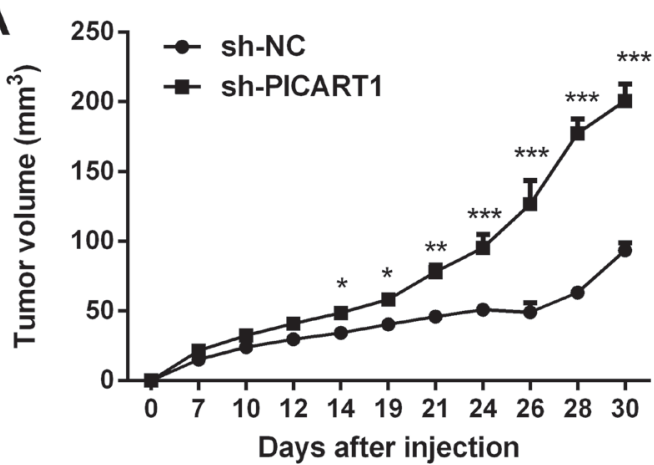

B

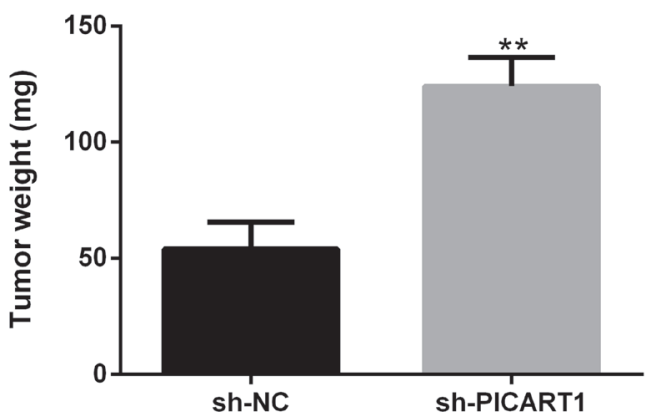

Figure 8. Effect of PICART1 knockdown on tumor formation in vivo. SPC-A-1 and NCI-H1975 cells were transfected with sh-PICART1 or sh-NC. A) After injection, tumor length and width was measured by a standard caliper, and the tumor volume was calculated by the formula: length $\times$ width ${ }^{2} \times$ 0.52. (B) After 30 days, the mice were killed by cervical dislocation, and the tumor weight was assessed.

growth by interacting with EZH2 and LSD1 and repressing LATS2 and KLF2 expression [24]; (2) LncRNA TATDN1 promotes NSCLC growth and metastasis by suppressing E-cadherin and Ezrin [25]; (3) lncRNA HIT functions as a pro-metastatic oncogene by directly associating with ZEB1 to regulate NSCLC migration, invasion and metastasis [23] and (4) ANRIL [7], PVT1 [22] and SNHG7 [26], PCAT1 [27] act as oncogenes in lung cancer progression.

In contrast, some lncRNAs act as tumor suppressors in lung cancer; (1) PANDAR, a direct transcriptional target of p53 in NSCLC cells, represses cell proliferation in vitro and in vivo by modulating $\mathrm{Bcl} 2$ expression [28]: (2) MEG3 expression is regulated by the retinoblastoma protein $(\mathrm{Rb})$ pathway. It is a lung cancer tumor suppressor through inhibition of cell proliferation and induction of apoptosis in A549 and SK-MES-1 cells [29] and (3) lncRNA PICART1 is reported to inhibit cell growth, proliferation, migration and invasion by regulating the AKT/GSK3 $\beta / \beta$-catenin signalling pathway in breast and colorectal cancer cells [17].

Despite this current research, PICART1's effect on lung cancer was not investigated before we established that it is down-regulated in lung tumor tissues and cell lines, and its over-expression reduces cell viability and induces cell cycle arrest in the G0/G1 phase and apoptosis. As seen in previous reports of PICART1's influence in breast and colorectal cancer cells, PICART1 reduces migration in SPC-A-1 and NCI-H1975 cells. This combined data indicates that PICART1 is a likely a tumor suppressor in lung cancer cells.

Many signaling pathways have important roles in lung cancer. These include the JAK2/STAT3 signaling pathway which is in various signaling cascades; including signaling tyrosine kinases and G-protein-coupled receptors [30]. Activation of the JAK/STAT signaling pathway is closely associated with cell proliferation and apoptosis, and the constitutive activation of both JAK2 and STAT3 is identified as crucial in the development of malignant transformation and tumorigenesis [31]. High expression levels of molecules involved in the JAK2/STAT3 signaling pathway are also associated with decreased lung cancer cell survival rate.

STAT3 is also a pivotal molecular target because of its powerful intervention in NSCLC anti-angiogenesis therapy [32]. Therefore, we explored if PICART1 regulates the JAK2/ 
STAT3 signaling pathway in the SPC-A-1 and NCI-H1975 cell lines. Our data confirms that PICART1 down-regulation promotes JAK2/STAT3 signaling pathway activation and its over-expression provokes the opposite effect.

We also determined that the Stattic blocked PICART1 knockdown effect on protein expression correlated with apoptosis and migration. This suggests PICART1 affects cell apoptosis and migration through regulation of the JAK2/ STAT3 pathway. Results of the in vivo tumor formation examined by xenograft tumor model assay revealed that PICART1 knockdown promotes tumor volume and weight. This further indicates PICART1's tumor suppressor role in lung cancer cells.

In conclusion, our study confirmed that PICART1 expression was remarkably down-regulated in lung cancer tissues; but its over-expression repressed cell proliferation and migration and induced cell apoptosis. Interestingly, the mechanism of PICART1 effect on cell growth is most likely by regulation of theJAK2/STAT3 signaling pathway. To the best of our knowledge, this is the first study demonstrating that PICART1 can be regarded as a tumor suppressor in lung cancer. This provides the new insight that PICART1 acts as a favourable progression indicator, and further clarification should confirm that it can be successfully used as a therapeutic target in future lung cancer patients.

Acknowledgments: This study was supported by the Postdoctoral Research Project of Qingdao (grant number 2016055) and Youth Foundation of the Affiliated Hospital of Qingdao University.

\section{References}

[1] MAJORES M, SCHINDLER A, FUCHS A, STEIN J, HEUKAMP L et al. Membranous CD24 expression as detected by the monoclonal antibody SWA11 is a prognostic marker in non-small cell lung cancer patients. BMC Clin Pathol 2015; 15: 19. https://doi.org/10.1186/s12907-015-0019-z

[2] CHEN HH, YAN JJ, CHEN WC, KUO MT, LAI YH et al. Predictive and prognostic value of human copper transporter 1 (hCtrl) in patients with stage III non-small-cell lung cancer receiving first-line platinum-based doublet chemotherapy. Lung Cancer 2012; 75: 228-234. https://doi. org/10.1016/j.lungcan.2011.06.011

[3] JEMAL A, SIEGEL R, XU J, WARD E. Cancer statistics, 2010. CA Cancer J Clin 2010; 60: 277-300. https://doi. org/10.3322/caac. 20073

[4] WANG XL, SHI WP, SHI HC, LU SC, WANG $\mathrm{K}$ et al. Knockdown of TRIM65 inhibits lung cancer cell proliferation, migration and invasion: A therapeutic target in human lung cancer. Oncotarget 2016; 7: 81527-81540. https://doi. org/10.18632/oncotarget.13131

[5] JONES PA, BAYLIN SB. The epigenomics of cancer. Cell 2007; 128: 683. https://doi.org/10.1016/j.cell.2007.01.029

[6] OMURA N, GOGGINS M. Epigenetics and epigenetic alterations in pancreatic cancer. Int J Clin Exp Pathol 2009; 2: 310-326.
[7] LIN L, GU ZT, CHEN WH, CAO KJ. Increased expression of the long non-coding RNA ANRIL promotes lung cancer cell metastasis and correlates with poor prognosis. Diagn Pathol 2015; 10: 14.

[8] WEI MM, ZHOU GB. Long Non-coding RNAs and Their Roles in Non-small-cell Lung Cancer. Genomics Proteomics Bioinformatics 2016; 14: 280-288. https://doi.org/10.1016/j. gpb.2016.03.007

[9] HU W, ALVAREZ-DOMINGUEZ JR, LODISH HF. Regulation of mammalian cell differentiation by long non-coding RNAs. EMBO Rep 2012; 13: 971-983. https://doi. org/10.1038/embor.2012.145

[10] GIBB EA, BROWN CJ, WAN LL. The functional role of long non-coding RNA in human carcinomas. Mol Cancer 2011; 10: 38. https://doi.org/10.1186/1476-4598-10-38

[11] LI H, YU B, LI J, SU L, YAN M et al. Overexpression of lncRNA H19 enhances carcinogenesis and metastasis of gastric cancer. Oncotarget 2014; 5: 2318-2329. https://doi. org/10.18632/oncotarget.1913

[12] YUAN SX, YANG F, YANG Y, TAO QF, ZHANG J et al. Long noncoding RNA associated with microvascular invasion in hepatocellular carcinoma promotes angiogenesis and serves as a predictor for hepatocellular carcinoma patients' poor recurrence-free survival after hepatectomy. Hepatology 2012; 56: 2231-2241. https://doi.org/10.1002/hep.25895

[13] ZHANG EB, YIN DD, SUN M, KONG R, LIU XH et al. P53regulated long non-coding RNA TUG1 affects cell proliferation in human non-small cell lung cancer, partly through epigenetically regulating HOXB7 expression. Cell Death Dis 2014; 5: e1243. https://doi.org/10.1038/cddis.2014.201

[14] ZHANG EB, KONG R, YIN DD, YOU LH, SUN M et al. Long noncoding RNA ANRIL indicates a poor prognosis of gastric cancer and promotes tumor growth by epigenetically silencing of miR-99a/miR-449a. Oncotarget 2014; 5: 22762292. https://doi.org/10.18632/oncotarget.1902

[15] ZHENG HT, SHI DB, WANG YW, LI XX, XU Y et al. High expression of lncRNA MALAT1 suggests a biomarker of poor prognosis in colorectal cancer. Int J Clin Exp Pathol 2014; 7: 3174-3181.

[16] CHAKRAVARTY D, SBONER A, NAIR SS, GIANNOPOULOU E, LI R et al. The oestrogen receptor alpha-regulated lncRNA NEAT1 is a critical modulator of prostate cancer. Nat Commun 2014; 5: 5383. https://doi.org/10.1038/ncomms6383

[17] CAO Y, LIN M, BU Y, LING H, HE Y et al. p53-inducible long non-coding RNA PICART1 mediates cancer cell proliferation and migration. Int J Oncol 2017; 50: 1671-1682. https://doi.org/10.3892/ijo.2017.3918

[18] JIANG C, XIN L, HUI Z, LIU H. Long non-coding RNAs: potential new biomarkers for predicting tumor invasion and metastasis. Mol Cancer 2016; 15: 62. https://doi.org/10.1186/ s12943-016-0545-z

[19] LEUNG A, NATARAJAN R. Noncoding RNAs in vascular disease. Curr Opin Cardiol 2014; 29: 199-206. https://doi. org/10.1097/HCO.0000000000000054 
[20] KOGO R, SHIMAMURA T, MIMORI K, KAWAHARA K, IMOTO $S$ et al. Long noncoding RNA HOTAIR regulates polycomb-dependent chromatin modification and is associated with poor prognosis in colorectal cancers. Cancer Res 2011; 71: 6320-6326. https://doi.org/10.1158/0008-5472. CAN-11-1021

[21] ZHOU Z, LIU H, WANG C, LU Q, HUANG Q et al. Long non-coding RNAs as novel expression signatures modulate DNA damage and repair in cadmium toxicology. Sci Rep 2015; 5: 15293. https://doi.org/10.1038/srep15293

[22] YANG YR, ZANG SZ, ZHONG CL, LI YX, ZHAO SS et al. Increased expression of the IncRNA PVT1 promotes tumorigenesis in non-small cell lung cancer. Int J Clin Experimental Pathol 2014; 7: 6929-6935.

[23] JIA X, WANG Z, QIU L, YANG Y, WANG Y et al. Upregulation of LncRNA-HIT promotes migration and invasion of non-small cell lung cancer cells by association withZEB1. Cancer Med 2016; 5: 3555-3563. https://doi.org/10.1002/ cam 4.948

[24] LI W, SUN M, ZANG C, MA P, HE J et al. Upregulated long non-coding RNA AGAP2-AS1 represses LATS2 and KLF2 expression through interacting with EZH2 and LSD1 in nonsmall-cell lung cancer cells. Cell Death Dis 2016; 7: e2225. https://doi.org/10.1038/cddis.2016.126

[25] NIU Z, ZHANG X, LI W, MING Z, ZHONG Y et al. The role and potentialmechanisms of LncRNA-TATDN1 on metastasis and invasion of non-small cell lung cancer. Oncotarget 2016; 7: 18219-18228. https://doi.org/10.18632/oncotarget.7788

[26] SHE K, HUANG J, ZHOU H, HUANG T, CHEN G et al. lncRNA-SNHG7 promotes the proliferation, migration and invasion and inhibits apoptosis of lung cancer cells by enhancing the FAIM2 expression. Oncol Rep 2016; 36: 26732680. https://doi.org/10.3892/or.2016.5105
[27] ZHAO B, HOU X, ZHAN H. Long non-coding RNA PCAT1 over-expression promotes proliferation and metastasis in non-small cell lung cancer cells. Int J Clin Exp Med 2015; 8: 18482-18487.

[28] HAN L, ZHANG EB, YIN DD, KONG R, XU TP et al. Low expression of long noncoding RNA PANDAR predicts a poor prognosis of non-small cell lung cancer and affects cell apoptosis by regulating Bcl-2. Cell Death Dis 2015; 6: e1665. https://doi.org/10.1038/cddis.2015.30

[29] KRUER TL, DOUGHERTY SM, REYNOLDS L, LONG E, DE SILVA $T$ et al. Expression of the lncRNA Maternally Expressed Gene 3 (MEG3) Contributes to the Control of Lung Cancer Cell Proliferation by the Rb Pathway. PLoS One 2016; 11: e0166363. https://doi.org/10.1371/journal. pone. 0166363

[30] PFEIFFER M, HARTMANN TN, LEICK M, CATUSSE J, SCHMITT-GRAEFF A et al. Alternative implication of CXCR4 in JAK2/STAT3 activation in small cell lung cancer. Br J Cancer 2009; 100: 1949-1956. https://doi.org/10.1038/ sj.bjc. 6605068

[31] LEVY DE, DARNELL JE JR. Stats: transcriptional control and biological impact. Nat Rev Mol Cell Biol 2002; 3: 651662. https://doi.org/10.1038/nrm909

[32] ZHAO M, GAO FH, WANG JY, LIU F, YUAN HH et al. JAK2/STAT3 signaling pathway activation mediates tumor angiogenesis by upregulation of VEGF and bFGF in nonsmall-cell lung cancer. Lung Cancer 2011; 73: 366-374. https://doi.org/10.1016/j.lungcan.2011.01.002 